\title{
The first 4 years - Outcome of children identified by newborn screening for $\mathrm{CF}$ in Germany
}

\author{
Katharina Schuetz ${ }^{1}$, Julia Kontsendorn ${ }^{2}$, Nils Janzen ${ }^{3}$, Jan Fuge ${ }^{2}$, Simon Grewendorf ${ }^{1}$, \\ Christian Klemann ${ }^{1}$, Christine Happle ${ }^{2}$, Sibylle Junge ${ }^{2}$, Isa Rudolf ${ }^{2}$, Christian Dopfer ${ }^{2}$, \\ Ludwig Sedlacek ${ }^{2}$, Diane Renz ${ }^{2}$, Gesine Hansen ${ }^{2}$, and Anna Maria Dittrich ${ }^{2}$ \\ ${ }^{1}$ Medizinische Hochschule Hannover \\ ${ }^{2}$ Hannover Medical School \\ ${ }^{3}$ Newborn Screening Laboratory
}

July 7,2021

\begin{abstract}
Background: Newborn screening (NBS) has been shown to improve CF disease course and has been widely implemented worldwide. This monocentric study compared children diagnosed by NBS vs. a cohort preceding the implementation of NBS in Germany in 2016 to evaluate ascribed benefits of NBS. Methods: We compared all children with confirmed CF diagnosis ( $\mathrm{n}=19$, "NBS group") out of all children presenting with positive NBS at our center after implementation of NBS (n=100) to children diagnosed with CF at our center within 4 years before NBS implementation (n=29, "pre-NBS group") for outcomes of anthropometry, gastrointestinal and pulmonary disease manifestations and respiratory microbiology. Results: Children diagnosed by NBS had a lower incidence of initial feeding problems (15\% vs. 41\%), had a higher BMI z-score at diagnosis and higher mean z-scores for BMI, weight and length during the study period. Children in the pre-NBS group displayed higher proportions of oxygen-dependent pulmonary exacerbations (10\% vs. $0 \%$ ), a significantly lower amount of normal bacterial flora $(\mathrm{p}=0.005)$ along with a significantly higher number of throat swab cultures positive for Pseudomonas aeruginosa $(\mathrm{p}=0.0154)$ in the first year of life. Yet, pulmonary imaging did not reveal less pulmonary morbidity in the NBS compared to the pre-NBS group. Conclusions: Our results confirm that NBS for CF leads to earlier diagnosis and improves nutritional outcomes in early childhood. Although trajectories of structural lung damage at early age were unaffected by NBS, NBS positive CF patients at preschool age displayed less severe pulmonary exacerbations and pathological bacteria in throat swabs.
\end{abstract}

\section{Hosted file}

Manuscript.doc available at https://authorea.com/users/424277/articles/529404-the-first-4years-outcome-of-children-identified-by-newborn-screening-for-cf-in-germany 
100 children NBS +

$\longrightarrow 3$ children: other CF centers

1 family refused sweat test

96: confirmation diagnostics at the

Hanover Medical School

- 80 children IRT path

- 16 children IRT/PAP/DNA path

$\rightarrow 73 \mathrm{CF}$ excluded by sweat test

$\checkmark$

- $23 \mathrm{CF}$

Excluded from analysis due to inclonclusive genetics: - 1 CF (initially inconclusive genetics)

NBS Group: - 2 CFTR-RD

19 CF children

Figure 1: Study group. IRT; immunoreactive trypsin, PAP; pancreatitis-associated protein; CFTR-RD; CFTR-related disease; CF-SPID; CF screening positive, indeterminate diagnosis
Control Group (pre-NBS Group):

$29 \mathrm{CF}$ children diagnosed

within 4 yrs. before NBS

implementation in our center 


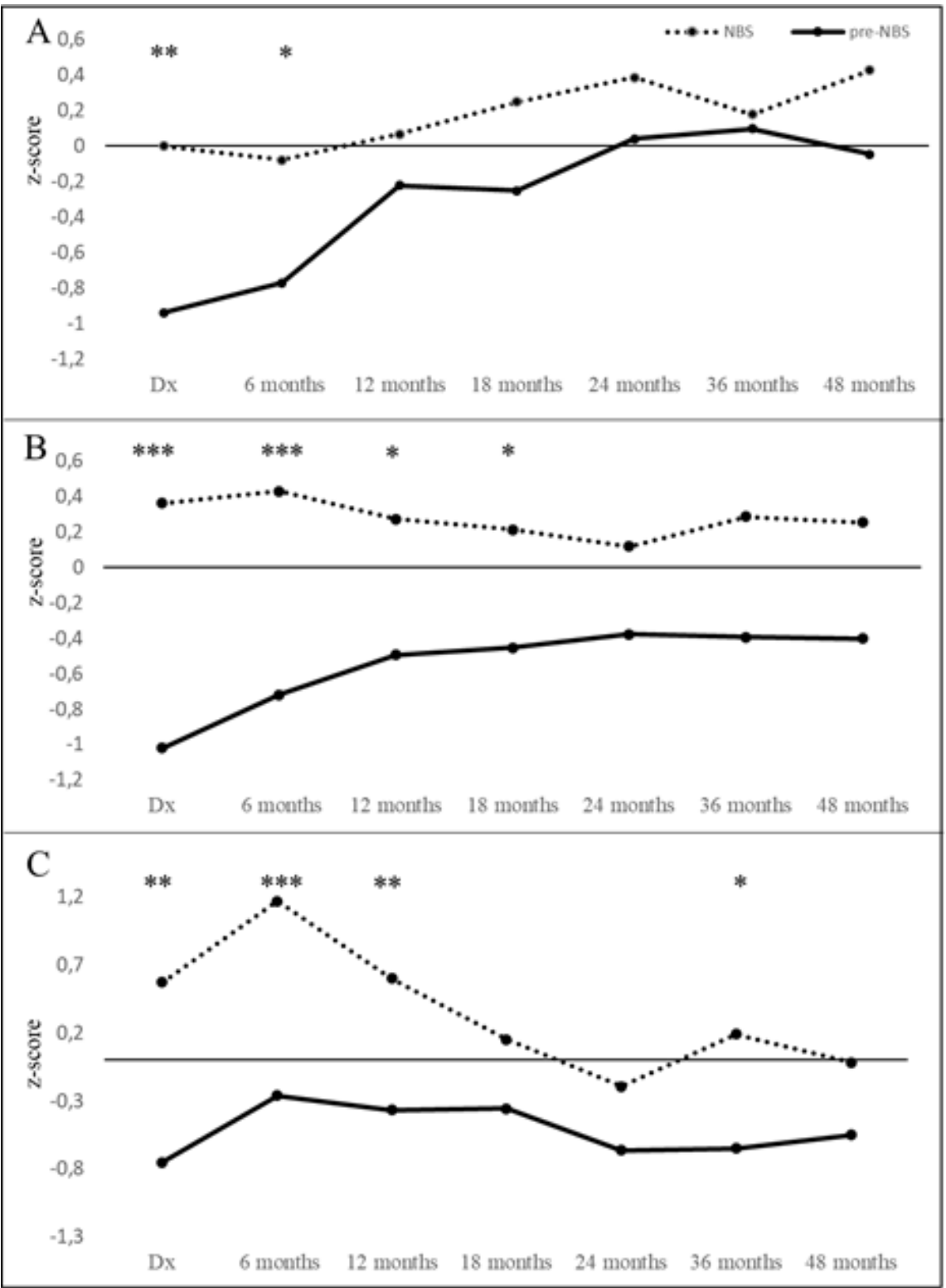

Figure 2: Anthropometric data over time for A: BMI, B: weight and C: length. X-Axis: Zscores of anthropometric values, Y-axis: age in months at analysis. Data were calculated by ttest in case of normal distribution and by Mann-Whitney $\mathrm{U}$ test in case of not normal distribution. $\mathrm{Dx}=$ diagnosis; NBS=Newbornscreening group; pre-NBS=pre-NBS group, * $\mathrm{p}<0.0 .05 ;{ }^{* *} \mathrm{p}<0.01,{ }^{* * *} \mathrm{p}<0.001$ 


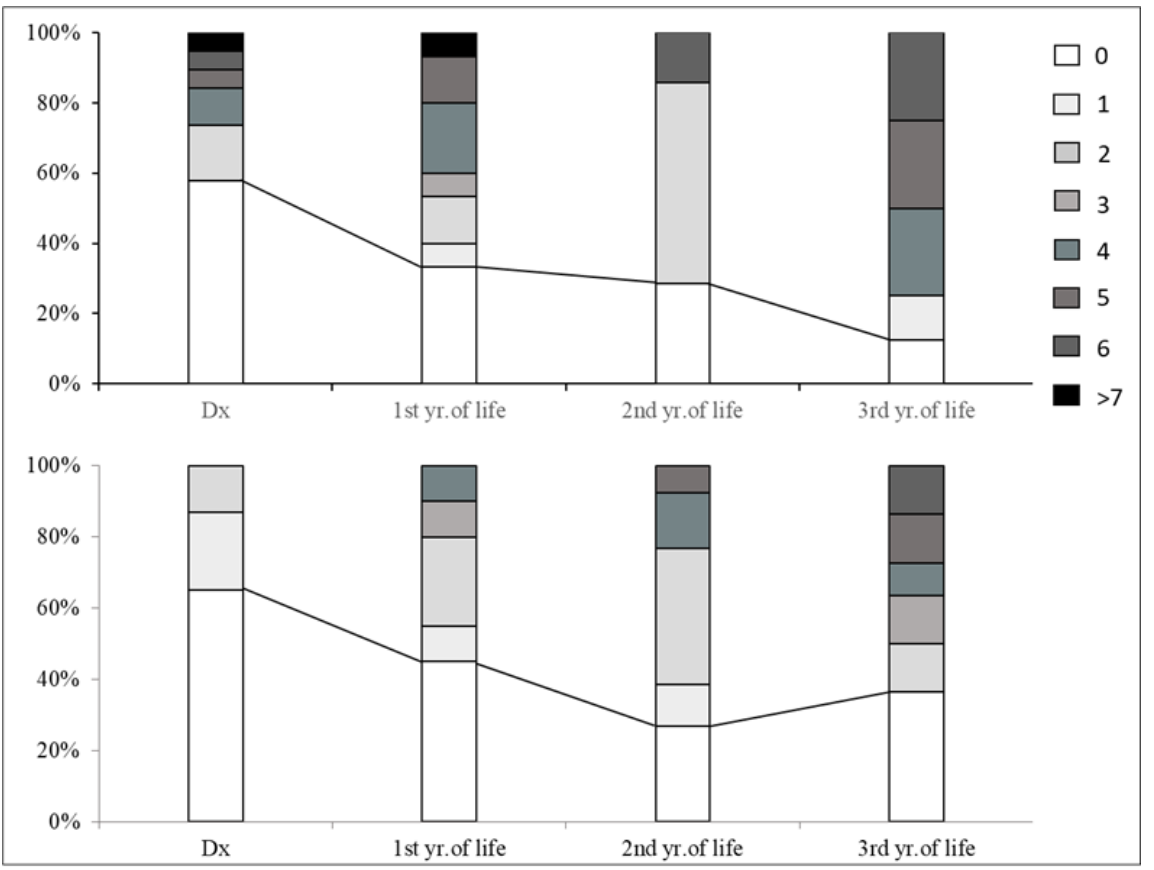

Figure 3: $\mathrm{mCN}$ scores over time in the A: NBS vs. B: pre-NBS group. Chest $\mathrm{x}$-ray images were conducted at time of diagnosis and annually thereafter. Scores were calculated according to a modified Crispin-Norman Score $(\mathrm{mCN})(11,12)$. X-Axis: life years, Y-axis: \% of children displaying designated $\mathrm{mCN}$-scores (see legend in Figure). Neither mean $\mathrm{mCN}$ scores nor $\%$ of children with specific mCN scores differed significantly between the NBS and the pre-NBS group. Data were not normally distributed and compared by Mann-Whitney U test. Dx= diagnosis, yr.= year 


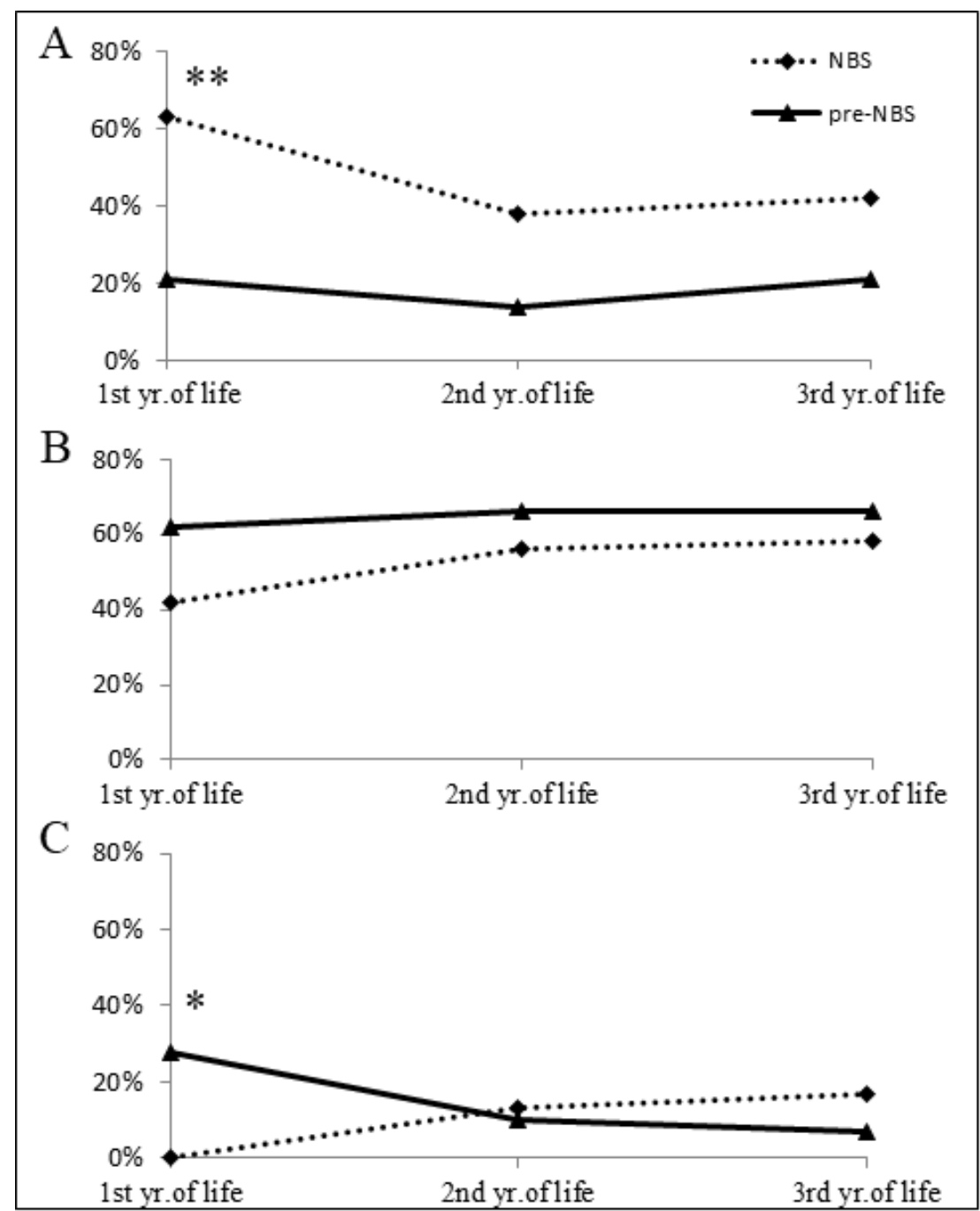

Figure 4: Throat swab microbiology over time in pre-NBS vs. NBS children. Frequency of children with detection of (A) normal flora, (B) $S A$ and (C) $P a$ per group. X-Axis: \% of children in group with positive throat swab cultures for respective flora/pathogen, $\mathrm{Y}$-axis: life years. Data were calculated by Fisher's exact test. NBS=Newbornscreening group; pre-NBS=pre-NBS group, yr.=year; ${ }^{*} \mathrm{p}<0.05$; $* * \mathrm{p}<0.01$

\section{Hosted file}

table 1.doc available at https://authorea.com/users/424277/articles/529404-the-first-4years-outcome-of-children-identified-by-newborn-screening-for-cf-in-germany 